POS $\quad$ PROCEEDINGS

\title{
Possible Precise Neutrino Unitarity?
}

\author{
Anatael Cabrera*† \\ CNRS-IN2P3 \\ LAL, Univ. Paris-Sud, CNRS/IN2P3, Université Paris-Saclay, Orsay, France \\ LNCA Underground Laboratory, CNRS/IN2P3 - CEA, Chooz, France \\ E-mail: anataelein2p3.fr
}

The exploration of the Standard Model (SM) leptonic mixing has been led by the study of the neutrino $(v)$ oscillations phenomenon, whose discovery was acknowledged by the 2015 Nobel prize in physics. Half a century of experimental and theoretical effort has established and demonstrated consistency with the $3 v$ model and with the so far SM three family evidence. While no direct significant manifestation for physics beyond the Standard Model (BSM) has been found, the SM is known not to suffice to explain all today's observed phenomenology. In the forthcoming decade, most oscillation parameters are expected to yield sub-percent precision. Such a knowledge opens the possibility to experimentally test for BSM manifestation(s) via the direct and competitive exploration of the PMNS matrix unitarity for the first time. Any significant deviation might, in turn, evidence the existence of non-standard states (i.e. new neutrino) and/or interactions, thus allowing for direct discovery potential. Even if no deviations were found, the PMNS matrix structure, very different from its CKM counterpart, is of fundamental importance to our understanding of the leptonic flavour sector. In this document, we shall briefly review today's PMNS unitarity status in the context of existing and future particle physics programme within the next decade. We identify the possible need for a missing experiment(s). One such a case maybe a hypothetical Super Chooz, employing the novel LiquidO technology, to address both directly sensitivity to the unitarity and unique impact to the exploration of the neutrino oscillation phenomena. Such a program is expected to additionally and coherently reinforce the physics of all currently planned experiments via indirect information aiding both the $\mathrm{CP}$ violation and mass ordering forthcoming measurements.

European Physical Society Conference on High Energy Physics - EPS-HEP2019 -

10-17 July, 2019

Ghent, Belgium

\footnotetext{
* Speaker.

${ }^{\dagger}$ The author would like thank the LiquidO collaboration for ongoing fruitful discussion.
} 
Today's neutrino oscillation experimental evidence is consistent with a $3 v$ framework [1]. This is in agreement with the observed three families of charged fermions making part of the Standard Model of Particle Physics (SM). While few inconclusive indications for possible discrepancy to the $3 v$ framework have been reported, intense exploration has cornered the remaining solution phasespace to marginal region(s) [2] - still not fully ruled out. Thus, unambiguous manifestation of physics beyond the Standard Model (BSM) remains elusive.

Since $v$ oscillation is the macroscopic manifestation of the quantum interference of neutrino mass states during their propagation and the mixing among mass $\left(v_{1}, v_{2}, v_{3}\right)$ and weak-flavour $\left(v_{e}\right.$, $v_{\mu}, v_{\tau}$ ) eigenstates, the entire phenomenon is characterised in terms of two mass squared difference $\left(\delta m^{2} \text { and } \Delta m^{2}\right)^{1}$ and three mixing angles $\left(\theta_{13}, \theta_{12}, \theta_{23}\right)$, embedded in the $3 \times 3$ PMNS mixing matrix. This is the CKM quark counterpart. This simplified parametrisation relies on a critical assumption: the PMNS matrix is unitary (labelled $U$ ). This same condition allows for a complex phase leading to $\mathrm{CP}$-violation ${ }^{2}(\mathbf{C P V})$ during mixing. There is no a priori prediction for any such parameters (6), so each must be measured to allow the phenomenological $3 v$ model characterisation of today's observations as well as possible searches for significant deviations between data and model, where discoveries may lay. It is worth noticing that the experimental test of the unitarity is as important as the measurement of their derived parameters. However, testing the unitarity implies addressing a larger system of equations, where the $\theta_{13}, \theta_{12}, \theta_{23}$ parametrisation no longer stands.

For about 50 years, the experimental community has been devoted to the measurement of each of the neutrino oscillation parameters. The key realisation was that behind the historically called solar and atmospheric anomalies, there is one single phenomenon: neutrino oscillation. However, the 2015 Nobel prize [3] discovery acknowledgement awaited the observation of the predicted new oscillation, driven by $\theta_{13}$. This was only significantly observed in 2011-2012 by Daya Bay (DYB) [4], Double Chooz (DC) [5] and RENO [6] experiments. Now we know neutrinos are massive even though we have not been able to measure its mass directly [7]. Today's knowledge can be effectively characterised by the precision of each parameters, since no significant deviations have been found, as summarised in Table 1 . While $\theta_{12}$ and $\theta_{23}$ are large, $\theta_{13}$ is very small. As of mid-2019, all parameters are known to the few percent $(<2.5 \%)$ upon combining all experiments data. Two major unknowns remain: atmospheric mass ordering ${ }^{3}$ and the CPV phase. There is preliminary evidence [1] amounting that a) normal mass ordering may be favoured at $\sim 3 \sigma$ 's and b) CP conservation may be disfavoured at $\sim 2 \sigma$ 's. Despite major success, today's precision is still limited to address the PMNS unitarity competitively; i.e. sub-percent level.

In the first half of 2020 decade, the sub-percent precision regime is expected. This will start with measurements of $\theta_{12}$ and $\delta m^{2}$ by JUNO [12], based in China. DUNE [13] and HK [14], based in USA and Japan, respectively, are expected to provide the ultimate knowledge on $\theta_{23}$ during the second half of the decade. The knowledge of $\Delta m^{2}$, including the mass ordering resolution, is expected to be led by both JUNO and DUNE using complementary vacuum and matter effects approaches, respectively. Surprisingly, no experiment is able to significantly improve today's $\theta_{13}$ precision $(1.5 \%)$, while all experiments depend strategically on it for both CPV and mass order-

\footnotetext{
${ }^{1} \delta m^{2}$ and $\Delta m^{2}$ provide notation for the so called "solar" $\left(\Delta m_{12}^{2}\right)$ and "atmospheric" $\left(\Delta m_{23}^{2}\right.$ or $\left.\Delta m_{13}^{2}\right)$ cases.

${ }^{2}$ The CPV implies different manifestation for matter and anti-matter, as observed in the 60's with quarks.

${ }^{3}$ This stands as the sign of $\Delta m^{2}$ unknown since mainly vacuum oscillation has been used to measure it. The sign of $\delta m^{2}$ is known due to matter dominated enhanced oscillations in the core of the sun.
} 


\begin{tabular}{|c|c|c|c|c|c|}
\hline & \multicolumn{2}{|c|}{ knowledge as of 2020 } & \multicolumn{3}{c|}{ expected knowledge beyond 2020 } \\
& dominant & precision $(\%)$ & precision $(\%)$ & dominant & technique \\
\hline$\theta_{12}$ & SNO & 2.3 & $\leq 1.0$ & JUNO & reactor \\
$\theta_{23}$ & NOvA & 2.0 & $\sim 1.0$ & DUNE+HK & beam \\
$\theta_{13}$ & DYB & 1.5 & 1.5 & DC+DYB+RENO & reactor \\
$\delta m^{2}$ & KL & 2.3 & $\leq 1.0$ & JUNO & reactor \\
$\left|\Delta m^{2}\right|$ & DYB+T2K & 1.3 & $\leq 1.0$ & JUNO+DUNE+HK & reactor + beam \\
\hline$\pm \Delta m^{2}$ & SK & unknown & measured?? & JUNO+DUNE+HK & reactor + beam \\
$\delta_{\mathrm{CP}}$ & T2K & unknown & measured?? & DUNE+HK & beam \\
\hline
\end{tabular}

Table 1: Neutrino Oscillation Knowledge. As of 2019, current and predicted knowledge on $3 v$ oscillation model is summarised in terms of the precision per parameter. The different columns show today's single experiment precision, dominant experiment, today's global precision (NuFit 4.0), predicted precision and best experiment along with the dominant technique used. The entire neutrino oscillation sector will be characterised using reactors and beams. This is not surprising since such man-made $v$ 's are best controlled in terms of baseline and systematics, as compared to atmospheric and solar $v$ 's. $\theta_{12}$ and $\theta_{23}$ will be largely improved by JUNO and DUNE+HK, respectively. JUNO will pioneer the sub-percent precision in the field. Interestingly, there is no foreseen capability to improve today's DC+DYB+RENO precision on $\theta_{13}$, whose knowledge will go from today's best to future worst, unless a dedicated experiment is proposed. $\delta m^{2}$ will be dominated by JUNO while $\Delta m^{2}$ will be constraints by both JUNO and DUNE+HK. The unknown mass ordering will be addressed mainly JUNO and DUNE using vacuum oscillations and matter effects, respectively. Global data analysis suggests a possible favoured normal ordering solution at $\sim 3 \sigma$ 's, dominated Super-Kamiokande [8] (SK) data. Any deviation between JUNO and DUNE would be of great interest. The unknown $\delta_{\mathrm{CP}}$ depends on DUNE+HK. Global data, dominated by T2K [9], disfavours CP conservation ( 0 or $\pi$ solutions) at $\sim 2 \sigma$ 's. Despite a key role in the field, the atmospheric neutrino experiments such as IceCube [10] and ORCA [11] are not expected to lead the ultimate precision by 2030, but intermediate results around 2025, if possible.

ing measurements. Our $\theta_{13}$ knowledge remains dominated by the aforementioned 2010 reactor data $[15,16,17]$. By 2030, only experiments relying on artificially produced neutrinos (i.e. reactors and beams) will dominate the ultimate neutrino oscillation knowledge, as described in Table 1. Thus, beyond 2020, the field is expected to be shaped by a few large (or huge) experiments with the highest budgets and largest (>500 scientists) collaboration per experiment in the history of neutrino research. It is worth noticing that no major neutrino oscillation experiment is envisaged to be based in Europe in the next decade.

In summary, upon the decade 2020-2030, the field will be reaching an overall sub-percent precision in all mixing angles, except for $\theta_{13}$. The unknown mass ordering and CPV are expected to be measured by 2030 with today's data already allowing some hinted solutions at a few $\sigma$ level. Hence, we will have all (6) parameters known by 2030. Since, arguably, it is difficult to imagine to go larger than JUNO+DUNE+HK experiments, so we must ensure that we are not missing anything compromising our ability to challenge the SM, thus maximising our best sensitivity to BSM possible manifestation(s), where discovery potential may be. This reflection is be addressed timely since each step in the field is currently implying decades (preparation and data-taking) and the subsequent resources. Indeed, this reflection is main the motivation of this document. 


\section{The PMNS Structure \& Unitarity}

This is one of the most critical questions to the field - arguably as important any consequent parameter. Let us consider the CPV phase for instances. It is interesting to note the CPV is expected within the neutrino oscillation framework (i.e. PMNS can be complex), while there is no established model behind the violation of unitarity. Instead, there is no SM prediction for the CPV phase value, while unitary enjoys an accurate prediction. Hence, the unitary exploration benefits from direct discovery potential in a model-less framework exploiting an accurate prediction to identify deviations. More, addressing unitary is complementary to today's measurements of each parameter, regardless of the overall PMNS structure. We shall below summarise, within the limitations of today's uncertainties, the main features of the PMNS matrix, as illustrated in Fig.1. Its structure offers some interesting features worth some intriguing questions:

Why is PMNS non-diagonal? Unlike the CKM, almost diagonal, thus leading to minimal mixing in quarks, the PMNS is largely non-diagonal. This means its "off-diagonal" terms are large, as shown in Fig 1. This implies that whatever BSM theory may stand behind the SM effective manifestation, the predicted flavour sector may be largely different for leptons and quarks.

It is striking to note that $\theta_{13}$ is the most peculiar, as it is very small and drives the value of $U_{e 3}$. Again, a possible hint from Nature suggesting that we ought to measure $\theta_{13}$ with the highest possible precision, as it might be key to understand the leptonic flavour sector. Ironically, no experiment today can improve 2010's results. Worse, there is up to now no experimental method known to be able to challenge those results. A new approach is highlighted below.

$$
\begin{aligned}
& \left(\begin{array}{ccc}
U_{e 1} & U_{e 2} & U_{e 3} \\
U_{\mu 1} & U_{\mu 2} & U_{\mu 3} \\
U_{\tau 1} & U_{\tau 2} & U_{\tau 3}
\end{array}\right) \approx\left(\begin{array}{c} 
\\
\end{array}\right) \\
& \text { electron row unitarity: }\left|U_{e 1}\right|^{2}+\left|U_{e 2}\right|^{2}+\left|U_{e 3}\right|^{2}=1
\end{aligned}
$$

Figure 1: The PMNS Neutrino Mixing Matrix. The non-diagonal structure and the smallness of the $U_{e 3}$ term (circled in red) are among the main features of the PMNS matrix, as illustrated. $U_{e 3}$ corresponds to $\theta_{13}$, if unitary. The overall PMNS unitarity test could be reduced to test the unitarity of the rows, where the most sensitive test today arises from the electron row (circled in blue).

Why is PMNS' $J$ so large? The PMNS Jarkslog invariant (factorising out the CPV phase $\sin (\delta)$ term) is order $\sim 10^{-2}$, which is much larger than that of the CKM counterpart $\left(\sim 10^{-5}\right)$. This suggests that if the CP was violated $(\sin \delta \neq 0)$, the expected CPV amplitude could be large. This is an appealing scenario as much CPV is needed to explain the observed matter to anti-matter asymmetry in the universe - orders of magnitude more compared to the CPV embedded in the CKM.

Is PMNS unitary? As highlighted above, this is likely to be the ultimate and most challenging question that the neutrino oscillation framework might allow us to explore. We shall address the possible implications and today's knowledge status below. 
The PMNS structure largely differs from the CKM. Hence, their nature may likely be different, although unknown. For some, the PMNS bizarreness (compared to CKM) might indicate that its most precise exploration and scrutiny is one of the best ways to challenge the SM. It would not be the first time neutrinos proved our best probe to BSM phenomenology. One of the latest modifications in the SM was the introduction of the phenomenology of massive neutrinos, as inferred from neutrino oscillations, although the absolute scale of their lightness remains a challenging mystery.

To address the PMNS unitarity, we need an overall sub-percent mixing precision. The results from JUNO, DUNE and HK are therefore critical. However, while necessary, those are not sufficient conditions to yield the deepest insight. Testing for the PMNS unitarity implies abandoning the three mixing angles $\left(\theta_{13}, \theta_{12}, \theta_{23}\right)$ approximation. Hence, the equations must be expressed in terms of their $U_{i j}$ terms upon imposing the unitary condition (i.e. $U U^{\dagger}=I$ ). This translates experimentally into constraining more equations. So, to test unitary to the percent level implies the need for the above described increase in precision but also additional measurements. This is described below. Indeed, only within the 2020 decade, the field is nearing a competitive level of precision. The reward of addressing this question is remarkable: any significant evidence for unitarity violation implies the manifestation, and thus discovery, of non-standard neutrino states and / or interactions [18]. Non-standard interactions (NSI) [19] stand for deviations during interaction and/or propagation of neutrinos. This implies direct sensitivity to BSM physics manifestation despite lacking the model behind. Given the stunning prediction power demonstrated by the SM to all so far tested observables, such those proved at the LHC, cosmology, etc; there is a diminishing phase space for direct access to discoveries in particle physics with today's technology. Hence, testing the PMNS unitary is indeed a compelling and unique opportunity.

\section{The PMNS Unitarity Test Strategy}

Solving the unitary condition $\left(U U^{\dagger}=I\right)$ leads to many equations [20]. Some are equivalent to testing the "closure of triangles", as practiced in the CKM case, should the CP violation be known. Since, the neutrino CPV phase is still unknown, the PMNS unitary condition can be tested today via the derived $\left|U_{l 1}\right|^{2}+\left|U_{l 2}\right|^{2}+\left|U_{l 3}\right|^{2}=1$ condition, with $l=e, \mu, \tau$. These equations test the unitarity of each matrix row. Only the $e$ and $\mu$ are considered since $\tau$ related oscillations are less constrained. In fact, the most stringent constraint arises from the electron-row unitarity (ERU) ${ }^{4}$ (or top row) leading to the $\left|U_{e 1}\right|^{2}+\left|U_{e 2}\right|^{2}+\left|U_{e 3}\right|^{2}=1$ accurate condition. If unitarity held, this row depends only on $\theta_{13}$ and $\theta_{12}$. Hence, any experiments with the ability to constrain $\theta_{13}$ and/or $\theta_{12}$ is of likely direct impact. ERU is today the only direct and most precise access to unitarity $[18,20]$.

This is excellent news for JUNO whose highest sensitivity to $\theta_{12}$ (and also $\delta m^{2}$ ) unprecedentedly grants some of the necessary sub-percent precision to test ERU. Indeed, JUNO is one of the most important experiment in the unitarity quest [12]. However, that is not good enough. Since, it is difficult to foresee any improvement on JUNO - even in the far future - we need other high precision measurements elsewhere. Unfortunately, the sensitivity on $\theta_{13}$ appears not improvable in foreseeable future. Only DUNE, at best, might reach a similar precision as of today. As discussed in $[18,20]$, testing for ERU implies several experimental constraints, here highlighted:

\footnotetext{
${ }^{4}$ The $\mu$-row case precision is limited by experimental uncertainties such the absolute flux (typically $<10 \%$ for beams), the unresolved atmospheric mass ordering and the "octant" ambiguity due to the almost maximal value of $\theta_{23}$.
} 
Via $\delta m^{2}$ Oscillations (i.e. $\theta_{12}$, if unitary): JUNO measures $P\left(\bar{v}_{e} \rightarrow \bar{v}_{e}\right)$ with reactor neutrinos over a $\sim 50 \mathrm{~km}$ baseline. Also, solar neutrinos have key information by probing $P\left(v_{e} \rightarrow v_{e}\right)$ in the core of the sun via matter effects. Today's best constraints come from SNO and SK. There is no dedicated solar experiment foreseen, although JUNO has some sensitivity.

Via $\Delta m^{2}$ Oscillations (i.e. $\theta_{13}$, if unitary): again reactor experiments, like DC and DYB, had measured $P\left(\bar{v}_{e} \rightarrow \bar{v}_{e}\right)$ at the baseline of order $1 \mathrm{~km}$. There is however no copious known $v_{e}$ source capable of addressing $P\left(v_{e} \rightarrow v_{e}\right)$ precisely enough with a compatible $\mathrm{L} / \mathrm{E}$ ratio.

Although not listed explicitly above, the absolute flux knowledge is of critical impact to test ERU $[18,20]$. However, the control of the absolute flux uncertainties is experimentally very challenging. This is indeed why many neutrino oscillation experiments use multi-detectors to bypass absolute systematics, as opposed to the simpler relative systematic basis. This way, for example, reactor experiments systematics can be controlled to the few per mille level while the absolute is controlled to order a few $\%$ at best. Worse, reactor neutrinos have evidenced a non-understood deficit [21] (2011) and spectral distorsion [22] (2014) relative to ILL-data based predictions. A hypothetical manifestation of non-standard neutrinos with $\Delta \mathrm{m}^{2}$ at $\sim 1 \mathrm{eV}^{2}$ had been considered. Today, however, such a hypothesis has lost much ground thanks to new data addressing this issue directly; i.e. weakening the hypothetical phase-space [2], and/or indirect; i.e. demonstrating that the reactor prediction uncertainties are likely larger [16]. Considering all those effects, today's studies [18, 20] quantify that the ERU test reach a few percent $(>2 \%)$ precision, including a prospected JUNO outcome. Hence, a dedicated experimental effort addressing the maximal sensitivity to unitarity is needed if a sub-percent precision level is to become possible - our goal for discussion here.

\section{Super Chooz Project Exploration}

Improving ERU test precision beyond JUNO requires (a) a significantly better measurement of $\theta_{13}$ (ideally sub-percent precision), (b) a much better control of absolute flux and, possibly, (c) a better measurement of solar neutrinos. Unfortunately, all those items are considered today either impractical - or even impossible - with today's technology.

However, a new neutrino detection technology called LiquidO [23] might allow to address some of those tough challenges. A hypothetical Super Chooz $(\mathbf{S C})$ project has been first raised in this HEP-EPS conference. The project would rely on an $\sim 10 \mathrm{kton}$ LiquidO detector located in one of the existing caverns upon the final deconstruction of the former Chooz-A reactor site. These caverns are to become available by $>2025$, implying minimal civil construction to reuse. This expansion implies that the existing LNCA laboratory (Chooz) would become one of the largest underground laboratories in Europe with two of the most powerful Areva N4 reactors as source despite low overburden ( $300 \mathrm{~m}$ water equivalent). While the physics potential is still under ongoing study, the performance depends on the LiquidO detection. The first experimental proof of principle [23] using its first opaque scintillator articulation [24] has been successfully demonstrated.

Super Chooz addressees the necessary to yield a $\theta_{13}$ measurement to $<1 \%$ precision - publication soon. This is a possible breakthrough since no technique so far is known to be able to reach such a precision. This precision could further aid enhance the sensitivities of DUNE and HK on $\mathrm{CPV}$, similar to today's reactor experiments have aided T2K sensitivity and possibly also JUNO's 


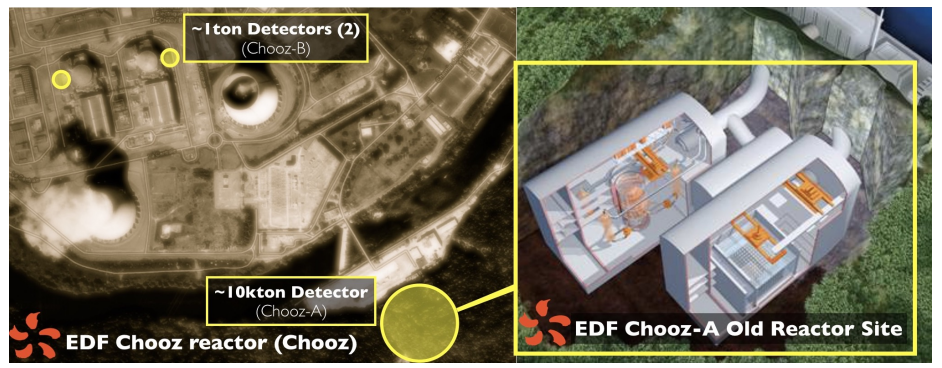

Figure 2: The Super Chooz Site. The SC experiment relies on two very-near detectors (order 1ton each) and one far large detector (order $10 \mathrm{kton}$ ). The multi-purpose far detector provide most of the physics programme (see text). The site relies on the scientific use of one of the old Chooz-A reactor caverns, provided by EDF, as an effective expansion of the existing LNCA laboratory.

mass ordering. A novel technique called reactor flux decomposition is needed to yield total reactor flux error cancellation, as the near detector technique, a la DC or DYB, are proved insufficient. Our preliminary studies suggest the world best precision on both $\theta_{13}$ and $\Delta m^{2}$ via shape extraction may be possible. Hence 2 (out of 6 ) of the parameters listed in Table 1 can be improved by Super Chooz, whose experimental configuration is shown in Fig 2. SC might be able to help the two other measurements needed for better unitarity precision absolute reactor flux and solar neutrinos, but this is under intense exploration still. Solar neutrino might benefit from an indium loading [23] to enable unprecedented solar neutrino measurement via CC interactions, unlike the more challenging electron elastic scattering. The main challenge here however is the control of cosmogenic backgrounds due to the lower overburden, while precise (mm scale) $\mu$ precise tracking might open for unprecedented tagging of the correlation between the primary $\mu$ and the spallation products.

Super Chooz could also become one of the best supernova neutrino (burst \& remnant) and proton decay detectors, while complementary to other foreseen detectors. On the supernova side, the ability for LiquidO to detect and identify $\bar{v}_{e}$ and $v_{e}$, upon CC interactions, allows unique capability for supernova neutrinos $(<50 \mathrm{MeV})$, including major background reduction and event-wise directionality. Flavour independent NC interaction detection is also possible upon loading, as highlighted in [23]. The supernova potential remains under active ongoing study. On the proton decay side, LiquidO's event-wise imaging, again, allows the event-wise identification of $\mathrm{K}^{+}, \pi^{0}, \pi^{ \pm}$, $\mu^{ \pm}$, etc. via their main decay mode(s). All of those particles play a role in different proton decay modes. LiquidO is expected thus to be one of the best proton decay searches technologies in terms of its highest free-proton density (normal in scintillators), high efficiency of detection and possible multi-decay mode sensitivity, boosted by its expected large background rejection. This was preliminary highlighted in [25], but further studies are ongoing.

The feasibility and vast physics programme potential of a hypothetical Super Chooz is under study within the LiquidO collaboration, supported by several other cooperating institutions. The Super Chooz project has the potential for unique breakthrough role in field, should LiquidO technology performance demonstrates. Its feasibility and full physics programme is expected to be clarified shortly. The main goal remains to articulate a programme to tackle all measurements needed to yield PMNS unitarity test with sub-percent precision. The success of this ambitious goal is under exploration but relies also on the precision to become available in the next decade. 


\section{References}

[1] NuFIT 4.1. [www.nu-fit.org.] (2019) JHEP 01106 (2019)

[2] Giunti C. et al. JHEP 1710143 (2017), Dentler M. et al. JHEP 10.1007010 (2018)

[3] SK Collab.(Fukuda Y. et al.) Phys.Rev.Lett. 81, 1562 (1998) SNO Collab.(Ahmad Q.R. et al.) Phys.Rev.Lett. 89, 011301 (2002) KamLAND Collab.(Eguchi K. et al.) Phys.Rev.Lett. 90, 021802 (2003)

[4] DYB Collab.(An F. et al.) Phys.Rev.Lett. 108171803 (2012)

[5] DC Collab.(Abe Y. et al.) Phys.Rev.Lett. 108, 131801 (2012)

[6] RENO Collab.(Ahn J. et al.) Phys.Rev.Lett. 108191802 (2012)

[7] Particle Data Group (Tanabashi M. et al.) Phys. Rev. D 98030001 (2018)

[8] SK Collab.(Jiang M. et al.) Preprint at arXiv: 1901.03230

[9] T2K Collab(Abe K. et al.) Phys. Rev. Lett. 118 15, 151801 (2017)

[10] IceCube Collab.(Aartsen M. G. et al.) Preprint at arXiv: 1401.2046

[11] ORCA Collab.(Adrián-Martínez S.) J.Phys. G43 8084001 (2016)

[12] JUNO Collab.(An F. P. et al.) J. Phys. G43 3030401 (2016)

[13] DUNE Collab.(Acciarri R et al.) Preprint at arXiv: 1512.06148

[14] HK Collab.(Abe K. et al.) PTEP 2015053 C02 (2015)

[15] DYB Collab.(An F. et al.) Phys.Rev.D 957072006 (2017)

[16] DC Collab.(de Kerret H et al.) Preprint at arXiv: 1901.09445

[17] RENO Collab.(Bak G. et al.) Phys.Rev.Lett. 121, 201801 (2018)

[18] Fong C. S. et al.. Preprint at arXiv: 1609.08623

[19] Y. Farzan and M. Tórtola. Preprint at arXiv: 1901.09445

[20] Qian X. et al.. Preprint at arXiv: 1308.5700

[21] Mueller T. et al. Phys.Rev.C 83054615 (2011) Huber P. Phys. Rev. C 85024617 (2011); 029901(E) (2012) Schreckenbach K. et al. Phys.Lett.B 160325 (1985) von Feilitzsch F. et al. Phys.Lett.B 118162 (1982) Hahn A. et al. Phys.Lett.B 218365 (1989)

[22] DC Collab.(Abe Y et al.) JHEP 10086 (2014) Erratum: JHEP 02074 (2015)

[23] Cabrera A et al.. LiquidO Preprint at arXiv: 1909.02859 (2019)

[24] Buck C. et al. Preprint at arXiv: 1908.03334

[25] Cabrera A et al. CERN Detector Seminar: LiquidO (2019) Web: https://indico.cern.ch/event/823865 